\title{
Ibuprofen Rescues Abnormalities in Periodontal Tissues in Conditional Presenilin 1 and Presenilin 2 Double Knockout Mice
}

\author{
Jiansheng Su ${ }^{1, \dagger, *}$, Jiamei Gu ${ }^{1, \dagger}$, Zhuo Dong ${ }^{2}$ and Bing Mei ${ }^{2, *}$
}

1 Laboratory of Oral Biomedical Science and Translational Medicine, School of Stomatology, Tongji University, Middle Yanchang Road 399, Shanghai 200072, China; E-Mail: jiamei.gu@live.cn

2 Shanghai Key Laboratory of Brain Functional Genomics,

Key Laboratory of Brain Functional Genomics, Ministry of Education, East China Normal University, Shanghai 200062, China;

E-Mail: dz820612@163.com

$\dagger$ These authors contributed equally to this work.

* Authors to whom correspondence should be addressed; E-Mails: sjs@tongji.edu.cn (J.S.); bmei@brain.ecnu.edu.cn (B.M.); Tel.: +86-21-6631-1629 (J.S.); +86-21-6223-3967 (B.M.); Fax: +86-21-6652-4025 (J.S.); +86-21-6260-1953 (B.M.).

Received: 17 June 2013; in revised form: 16 August 2013 / Accepted: 26 August 2013 /

Published: 6 September 2013

\begin{abstract}
We used forebrain-specific conditional presenilin 1 (PS1) and presenilin 2 (PS2) double knockout mice (dKO mice) that exhibit symptoms of neurodegenerative diseases, especially Alzheimer's disease, to investigate whether ibuprofen can rescue brain and periodontal tissue abnormalities by attenuating the inflammatory response. Mandibles were dissected for alveolar bone-height analysis. Maxillae were fixed and decalcified for histological observation and osteoclast detection. ELISA measurements from the hippocampus, cortex, and gingiva of the mandibular incisor teeth were used to assay inflammatory mediators. We confirmed periodontal tissue abnormalities and inflammatory responses in brain and periodontal tissues in naive nine- and 12-month-old $\mathrm{dKO}$ mice. The other two groups of age-matched $\mathrm{dKO}$ mice that received 375-ppm ibuprofen treatment for six consecutive months exhibited significantly attenuated damage in periodontal tissues and reduction in several inflammation-related factors in brain and periodontal tissues. Our findings showed that the anti-inflammatory drug ibuprofen significantly decreased
\end{abstract}


inflammation through the cyclooxygenase (COX) pathway in brain and periodontal tissues in $\mathrm{dKO}$ mice, and then attenuated abnormalities in periodontal tissues. This suggests that ibuprofen could be an ideal drug for preventing both nervous system and periodontal tissue damage caused by inflammatory responses.

Keywords: ibuprofen; presenilins; inflammatory response; periodontal tissues

\section{Introduction}

Presenilin mutations are linked to more than $90 \%$ of early onset familial Alzheimer's disease (AD) cases [1]. PS1-knockout mice are embryonic lethal, while forebrain-specific PS1-knockout mice are almost normal, but lack enrichment-induced neurogenesis [2]. PS2-knockout mice however, are viable, fertile, and exhibit no obvious abnormal phenotypes [3]. Mice lacking both PS1 and PS2 in the forebrain ( $\mathrm{dKO}$ mice) exhibit a series of age-dependent AD-like phenotypes, which include synaptic dysfunction, hyperphosphorylation of tau, and severe neurodegeneration [4-9], yet the level of $A \beta$ (amyloid $\beta$ ) deposits are significantly reduced [8]. In terms of cognitive ability, mild spatial and contextual memory impairment emerges as early as two months and continues to progress, reaching quite serious levels when the $\mathrm{dKO}$ mice reach six months [5]. Besides these typical hallmarks, inflammatory changes also present in the $\mathrm{dKO}$ brain as an early and prominent feature $[7,8]$. Another group in our lab has confirmed that microglia and astrocytes are dramatically activated and complement $\mathrm{C} 1 \mathrm{q} \alpha$ and $\mathrm{C} 4$ are stimulated from an early age (three months) in the dKO brain. In addition, leukocyte serum levels were reported to be elevated beginning at six months, and cytokine and chemokine levels, such as those of TNF- $\alpha$ and IL-1 $\beta$, were significantly higher both in brain and serum at nine months. These findings indicate that a robust inflammatory response appears early in the brains of $\mathrm{dKO}$ mice and then expands to the periphery [7].

Periodontitis is a lifelong, highly prevalent, peripherally infectious disease that is associated with gram-negative, anaerobic bacteria and is linked to localized and systemic inflammation. Gingival bleeding, loss of periodontal attachment and teeth are major markers of periodontitis [10]. Loss of periodontal attachment results in a decrease of alveolar bone height and exposure of the root surface [11]. Clinical studies have investigated the mutual influence between periodontitis and $A D$, and recent published studies show that elder adults with periodontitis or with several missing teeth exhibit more cognitive impairment compared with those without periodontitis or with few missing teeth [12-15]. According to other studies, poor cognition or cognitive decline may also lead to oral health issues such as tooth loss, caries, and poor plaque control [16-18]. Based on studies of dKO mice, it has been found that inflammatory responses in $\mathrm{dKO}$ mice brains can expand to oral tissue. The number of osteoclasts and amount of alveolar bone loss significantly increased at nine months, periodontal tissues showed obvious histomorphological abnormalities, and moreover, inflammatory mediators - such as TNF- $\alpha$ and IL-1 $\beta$-increased in gingiva with age. These data indicate that inflammation in $\mathrm{dKO}$ mice brains can expand to the periphery and finally lead to abnormalities in periodontal tissues [19].

Novel clinical and biological evidence has engendered renewed interest regarding strategies targeting the inflammatory processes and, in particular, on the potential use of non-steroidal 
anti-inflammatory drugs (NSAIDs) in preventing or treating AD. There is compelling epidemiological evidence that long-term NSAID therapy delays the onset of AD [20] and reduces one's lifetime risk of developing AD [21,22]. In addition, numerous experimental and clinical studies suggest NSAIDs as a therapeutic possibility for preventing alveolar bone resorption as periodontitis progresses [23]. Ibuprofen was one of the most frequently used anti-inflammatory drugs in those studies, and it may reduce the severity of inflammatory responses both in periodontitis and AD because it can cross the blood-brain barrier [21,23]. Here, we therefore examined whether six months of ibuprofen treatment could suppress inflammatory responses in the brain at three months, when inflammatory responses first appear in the $P S-\mathrm{dKO}$ mice, and at six months, when inflammatory responses expand to the periodontal tissue. We administered ibuprofen for six months because research indicates that reductions in IL-1 $\beta$ and insoluble $A \beta$ depend on the treatment duration (6 months $>3$ months) $[24,25]$. Moreover, this allows us to discuss potential therapeutic methods that might attenuate abnormalities in the brain and periodontal tissues.

\section{Results and Discussion}

\subsection{Ibuprofen Decreased Alveolar Bone Loss of the Mandibular Molar Region in PS-dKO Mice}

The periodontal attachment loss is a major marker of periodontal destruction, but it is difficult to quantify the width of the periodontal membrane. However, decrease in alveolar bone height and exposure of the root surface as a result of periodontal attachment loss are easily quantified [10]. The development of alveolar bone loss in naive $\mathrm{dKO}$ mice was much faster than in control mice (Figure 1A). Further, although the amount of root exposure in naive and ibuprofen-treated dKO mice did not significantly differ at nine months, at 12 months it was significantly reduced $(p<0.05)$ in naive dKO mice (Figure 1B).

Figure 1. Alveolar bone loss (A) Mandibular lingual aspects of control mice at nine and 12 months of age (9 months $n=7,12$ months $n=9$ ), naive double knockout mice (dKO mice) $(n=9)$, and ibuprofen-treated dKO mice (9 months $n=8,12$ months $n=9$ ). The area enclosed by the red line is the exposed molar root area; (B) Root exposure area $\left(\mathrm{mm}^{2}\right)$ of control, naive $\mathrm{dKO}$, and ibuprofen-treated $\mathrm{dKO}$ mice at nine and 12 months of age. Values are mean $\pm \mathrm{SE}$ for each group. ${ }^{*} p<0.05, * * p<0.01, * * * p<0.001$.
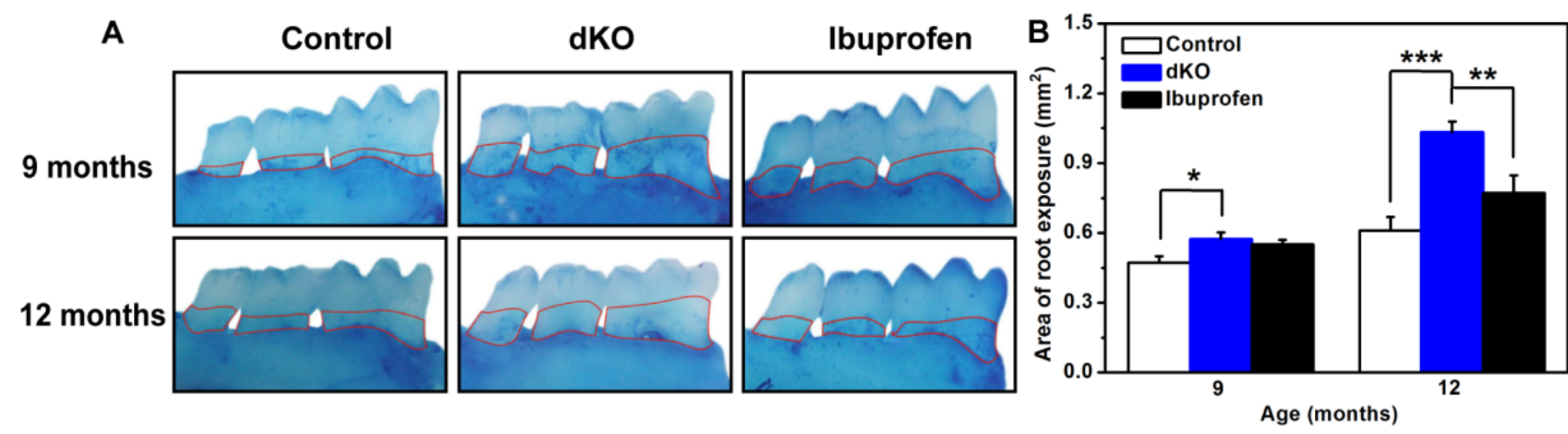


\subsection{The Number of Osteoclasts in the Periodontal Ligament Was Significantly Less in}

\section{Ibuprofen-Treated Mice}

The loss of connective tissue and bone in periodontal tissue is largely caused by the activation of osteoclasts and matrix metalloproteinases [10]. Osteoclasts differentiate from macrophage precursor cells in periodontal ligaments to absorb bone [26]. Therefore, we counted the number of osteoclasts (CTR-positive cells) in the periodontal ligament (Figure 2A,B) and found significantly more $(p<0.05)$ osteoclasts in naive $\mathrm{dKO}$ mice at both nine and 12 months compared with control and ibuprofen-treated dKO mice (Figure 2C).

Figure 2. Representative examples of immunostaining of osteoclasts in periodontal ligaments of naive dKO mice. (A) Negative control for CTR immunostaining in periodontal ligament; (B) CTR-positive osteoclasts and resorption lacunae in periodontal tissue. Magnification for A and B is 400×; (C) The number of osteoclasts. CTR-positive cells in the periodontal ligament from five sections of each animal were counted. Values are mean \pm SE. There were a significantly greater number of osteoclasts (CTR-positive cells) in naive dKO mice $(n=9)$ compared with control mice $(n=7-9)$ and ibuprofen-treated dKO mice $(n=8-9)$. * $p<0.05, * * p<0.01, * * * p<0.001$.

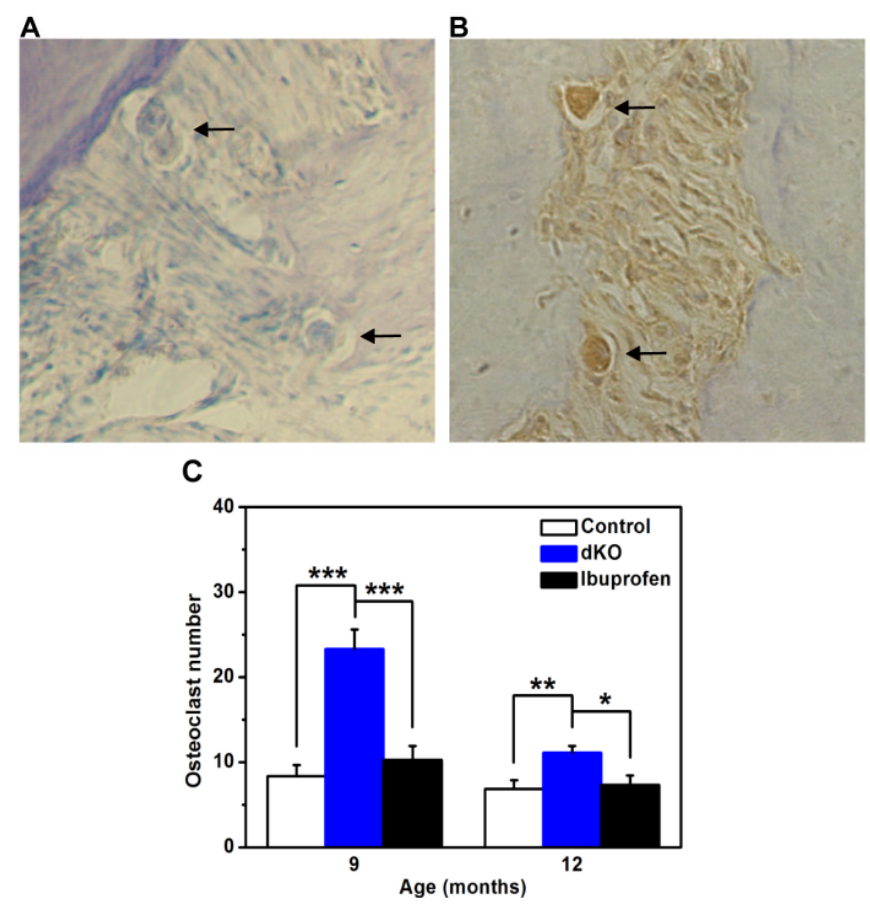

\subsection{Ibuprofen Alleviated Histomorphological Abnormalities in the Periodontal Tissue of dKO Mice}

In periodontitis, the inflammatory process extends from the gingiva to deeper connective tissues, resulting in the loss of connective tissue, bone, and finally teeth. Mild cementum and alveolar bone resorption causes an irregular increase in the width of the periodontal ligament due to an uneven reduction in the cortical plate in the bone and cementum layer [10]. In this study, periodontal ligament width in nine-month-old control mice was consistent and the cementum and alveolar bone was arranged normally. In contrast, periodontal ligament width in 12-month-old naive dKO mice was 
larger, the fibers of the periodontal ligament were fewer, and the cementum became irregular (Figure 3, white arrows). Inflammatory factors invaded the root of first molar (Figure 3, black arrows). After treatment with ibuprofen for six months, the abnormalities in periodontal tissue were partially absent (Figure 3F).

Figure 3. Histological observation of the periodontium (HE stained, 40× and 100×) Histological observations of control mice showed normal periodontal tissue structure (A,D); In naive dKO mice, periodontal tissues were infiltrated with inflammatory cells (black arrows) and the cementum became irregular (white arrow) (B,E); These abnormalities were partially absent after ibuprofen treatment $(\mathbf{C}, \mathbf{F})$.

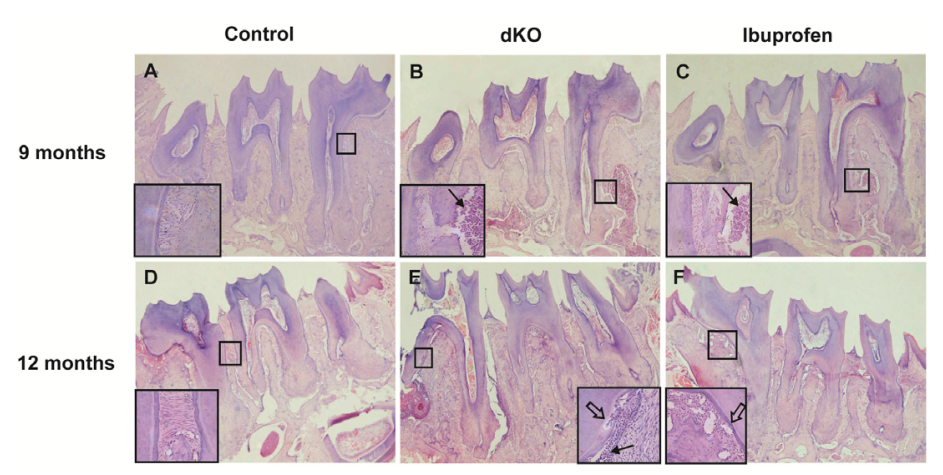

\subsection{Inflammatory Mediators in the Brain and Gingiva Were Reduced with Ibuprofen Treatment}

IL-1 $\beta$ and TNF- $\alpha$ levels in the gingiva, hippocampus, and cortex of naive dKO mice were significantly higher compared with nine- and 12-month-old control mice (Figure 4A-D). These levels were dramatically lower in the ibuprofen-treated group (Figure 4A-D). Noteworthy, average IL-1 $\beta$ levels in gingiva were significantly higher in the ibuprofen-treated group compared with the control group at nine months (Figure 4A). The results were normalized to wet tissue weight.

Figure 4. ELISA measurements of IL-1 $\beta(\mathbf{A}, \mathbf{B})$ and TNF- $\alpha(\mathbf{C}, \mathbf{D})$ from control, naive $\mathrm{dKO}$, and ibuprofen-treated $\mathrm{dKO}$ mice at nine and 12 months. $(\mathbf{A}, \mathbf{C})$ Gingiva of the incisor teeth. (B,D) Hippocampus and cortex. $* p<0.05, * * p<0.01, * * * p<0.001$.
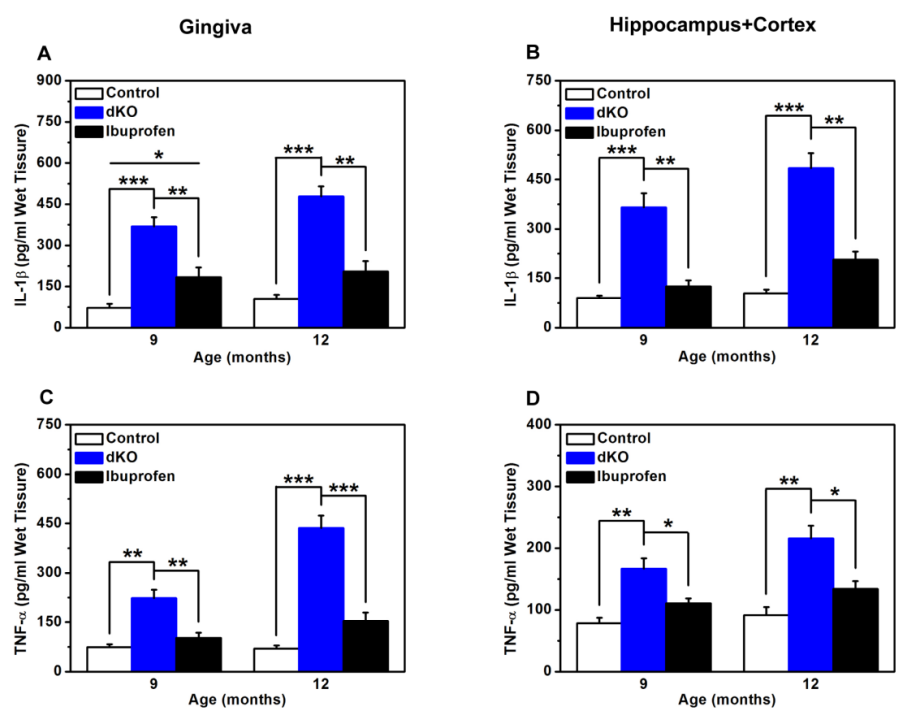


\subsection{COX-2 and PGE2 Levels Were Significantly Lower after Ibuprofen Treatment}

At both nine and 12 months, COX-2 and PGE2 levels were significantly higher in naive dKO mice compared with control mice (Figure 5A-D). In the ibuprofen-treated group, both COX-2 and PGE2 levels were dramatically lower than in the naive dKO group (Figure 5A-D). However, average COX-2 and PGE2 levels were significantly higher in the ibuprofen-treated group than in the control group at 12 months (Figure 5A-D).

Figure 5. ELISA measurements of COX-2 (A,B) and PGE2 (C,D) in control, naive dKO, and ibuprofen-treated dKO mice at nine and 12 months. (A,C) Gingiva of the incisor teeth. (B,D) Hippocampus and cortex. ${ }^{*} p<0.05, * * p<0.01, * * * p<0.001$.
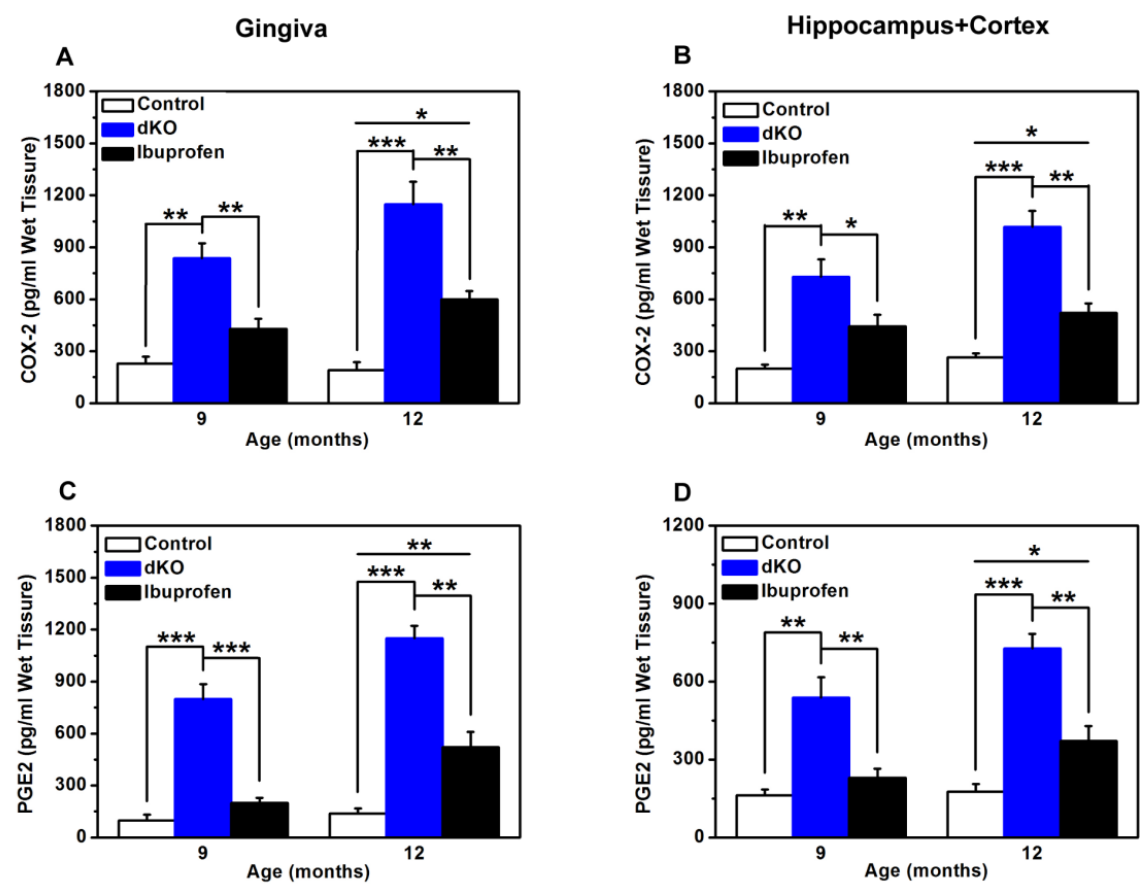

Our colleagues have found that the inflammatory response appeared in $P S \mathrm{dKO}$ mice at three months [7] and then spread to the periphery at six months, including the oral tissues, which can ultimately induce abnormalities in periodontal tissues [19]. Periodontal tissues (tooth-supporting tissues) include the gingiva, alveolar bone, cementum, and periodontal ligament. The periodontal ligament is located between the alveolar bone and the cementum [27]. The inflammatory process of periodontitis extends from the gingiva to deeper connective tissues, and severe resorption leads to tooth loss. Mild resorption of cementum and alveolar bone results in an irregular increase in the width of the periodontal ligament because the cortical plate of the alveolar bone and the cementum layer decrease unevenly [27]. In this study, histological observations of control mice showed normal periodontal tissue structure, while in naive $\mathrm{dKO}$ mice the periodontal ligament was irregular and thick, and the cortical plate and cementum layer were inconsistent and irregular (Figure 3). Alveolar bone height decreased with age in both control and naive $\mathrm{dKO}$ mice. However, when compared with control mice, the rate of alveolar bone loss in naive dKO mice was much faster and more severe (Figure 1). Furthermore, we found a significantly higher number of osteoclasts in the periodontal ligament of naive $\mathrm{dKO}$ mice than in control mice at nine and 12 months (Figure 2C). These results verified the 
periodontal tissue abnormalities in naive $\mathrm{dKO}$ mice found in our previous studies. As mentioned in the introduction, people with impaired cognition might be inattentive to oral hygiene or oral health maintenance, which results in poorer dental health [28]. Since no mice received oral care treatment in this study, our results indicate that inflammatory response is the most likely cause of the periodontal tissue abnormalities found in $\mathrm{dKO}$ mice.

In studies regarding the progression of periodontitis, ibuprofen has been one of the most frequently used anti-inflammatory drugs [23]. In this study, three-month-old and six-month-old dKO mice were fed chow that contained ibuprofen $(375 \mathrm{ppm})$ for six months. We found that ibuprofen alleviated the abnormalities in periodontal tissues: the number of inflammatory cells in the periodontal ligament was less and the cementum layer was consistent and regular (Figure 3), and alveolar bone loss was significantly less at 12 months (Figure 1C). Moreover, significantly fewer osteoclasts were found at nine and 12 months (Figure 2C), which was caused by less alveolar bone loss and a thinner periodontal ligament. Note, however, that here fewer osteoclasts is not an indication of alveolar bone loss because we only counted osteoclasts in periodontal ligaments, the area of which decreased substantially due to severe periodontitis. Periodontal diseases are most often caused by the interaction between specific bacteria and components of the host immune response, while the inflammation found in $\mathrm{dKO}$ mice is the result of infection. These findings support the idea that periodontal abnormalities in naive dKO mice are due to the severe inflammatory response that originates from brain and subsequently spreads to the periphery, and that long-term ibuprofen treatment could markedly alleviate these abnormalities through its anti-inflammatory effects.

Our colleagues have conducted another study in which ibuprofen inhibited the activation of microglia which had caused release of inflammatory cytokines such as IL- $1 \beta$ and TNF- $\alpha$ in the brain of naive dKO mice [29]. Transgenic mice over-expressing TNF- $\alpha$, or its receptors, in the brain develop chronic inflammation and neurodegeneration [30]. IL-1 $\beta$ has been reported to increase the expression of amyloid precursor protein (APP) in neuronal culture [31], and exposure of primary neurons to IL-1 $\beta$ exacerbates tau phosphorylation [32]. Therefore, we checked for IL-1 $\beta$ and TNF- $\alpha$ to assess the inflammatory response in naive $\mathrm{dKO}$ mice. Our results showed that levels of these inflammatory cytokines were higher not only in the brain but also in periodontal tissues in naive dKO mice at nine and 12 months (Figure 4), further confirming that periodontal tissue abnormalities resulted primarily from inflammatory responses in $\mathrm{dKO}$ mice.

Non-steroidal anti-inflammatory drugs (NSAIDs) have received considerable attention as a potential therapy both for preventing alveolar bone resorption in the progression of periodontitis [23] and for $\mathrm{AD}[22,33]$. NSAIDs are typically used to reduce inflammatory response by inhibiting COX isoforms. COX-1 is constitutively expressed in most tissues and generates protective prostanoids that are pro-inflammatory and that maintain the gastric mucosa. Expression of COX-2 is commonly induced with growth factors, tumor promoters, hormones, bacterial endotoxin, cytokines, anoxia, neurotoxins, electrical stimulation, and pro-inflammatory stimuli [34,35]. Therefore, it has been accepted that COX-1 is constitutively expressed whereas COX-2 is expressed as part of the inflammatory response in most tissues. Being the prostanoid most generally associated with inflammatory responses, the formation of prostaglandin E2 (PGE2) at inflammation sites is often taken as an indicator of local COX activity and its inhibition as an index of the ability of NSAIDs or newer COX-2-selective agents to inhibit COX-2. Ibuprofen, as one of the most frequently used NSAIDs, may 
reduce the severity of inflammatory responses in both periodontitis and $\mathrm{AD}[23,36]$. In our study, $\mathrm{dKO}$ mice treated with ibuprofen showed significantly lower levels of COX-2 and its metabolite PGE2 in the brain and periodontal tissue compared with non-treated dKO mice (Figure 5). These data confirmed that ibuprofen likely has a role in ameliorating both CNS inflammation and peripheral inflammation.

Based on their data, Akiyama and Hoozemans have suggested that NSAID-mediated COX inhibition might reduce inflammation in vivo by interrupting a putative feed-forward inflammatory mechanism [37,38]. Primary cell-culture experiments have shown that pro-inflammatory cytokines, such as IL- $1 \beta$ and TNF- $\alpha$, can up-regulate COX-2 expression and PGE2 in neurons, astrocytes, and microglia. Conversely, enhanced expression of COX-2 and PGE2 can increase the production of cytokines, prostanoids, and mediators of oxidative stress [37,38]. Our data showed a significant increase of COX-2 and PGE2 in naive dKO mice. Results proved that the pro-inflammatory cytokines IL-1 $\beta$ and TNF- $\alpha$ induced increases in the pro-inflammatory molecule COX-2 and its metabolite PGE2. We therefore examined the level of IL-1 $\beta$ and TNF- $\alpha$ in the brain and periodontal tissue. Compared with those in naive dKO mice, the levels of IL-1 $\beta$ and TNF- $\alpha$ were significantly lower in the brain and periodontal tissue of ibuprofen-treated mice. These results proved that reduction of inflammation caused by NSAID-mediated COX inhibition is through the putative feed-forward inflammatory mechanism.

Our data show that ibuprofen attenuated inflammation in the brain and in periodontal tissue in naive $\mathrm{dKO}$ mice at nine and 12 months. As mentioned above, a relatively straightforward argument was made for cognitive impairment leading to poor dental health: People with impaired cognition might be inattentive to oral hygiene or oral health maintenance as impairment in cognition progresses [28]. Our colleagues have found that ibuprofen partly alleviates learning and memory deficits in dKO mice [29]. We deduce that ibuprofen treatment might improve impaired cognition, including maintenance of good oral habits that is beneficial to oral hygiene and prevention of periodontitis. Moreover, reduction of brain inflammation via ibuprofen might help to decrease peripheral inflammation, such as periodontitis. In conclusion, ibuprofen could attenuate brain and periodontal inflammation, and these improvements might interact with each other to form a virtuous cycle.

\section{Experimental Section}

\subsection{Mice Breeding and Genotyping}

Breeding and genotyping of presenilins $(P S)$ dKO mice have been described previously [5,6,39]. Briefly, conditional PS dKO mice were obtained by crossing forebrain-specific PS1 heterozygous knockout mice with conventional PS2 heterozygous knockout mice on a B6/CBA F1 genetic background. Mice with the Cre transgene, fPS1/fPS1 and $P S 2^{-/-}$served as $\mathrm{dKO}$, their littermates (no Cre, $\mathrm{fPS}^{-/+}$and $P S 2^{+/+}$or $\mathrm{fPS1} 1^{-/+}$and $P S 2^{+/-}$) served as controls. dKO mice were fed either standard chow (naive $\mathrm{dKO}$ ) or standard chow containing $375 \mathrm{ppm}$ ibuprofen (ibuprofen-treated), the same dosage that has been used in previous studies of AD-model mice [24]. Treatment of the mice started at three and six months of age and lasted for six months, generating the nine and 12-month-old groups, respectively. All mice were housed at $20-26{ }^{\circ} \mathrm{C}$ and in $40 \%-70 \%$ humidity with $12 \mathrm{~h}$ 
light/dark cycles, and were sacrificed at the age of nine and 12 months. All experiments were approved by the Animal Ethics Committee of East China Normal University, China.

\subsection{Immunohistochemical Analysis of the Calcitonin Receptor in the Periodontal Ligament}

Immunohistochemical analysis was conducted following methods described in Han et al. [19]. Sections $(5 \mu \mathrm{m})$ of the upper molars were mounted onto amino-propyl-tri-ethoxy-silane (APES)-coated slides (Shanghai Biochip, Shanghai, China). After dewaxing in xylene, sections were dehydrated in ethanol and rinsed in distilled water for $10 \mathrm{~min}$. Antigen retrieval was performed by immersing slides in $0.1 \%$ trypsin (BIOS, Beijing, China) and 0.01 M Tris-buffered saline (TBS, pH 7.2) for $30 \mathrm{~min}$, and endogenous peroxidase activity was blocked with $3 \% v / v \mathrm{H}_{2} \mathrm{O}_{2}$ in water for $3 \mathrm{~min}$. For calcitonin receptor (CTR) detection, sections were incubated with a rabbit polyclonal anti-CTR primary antibody (bs-0124R, BIOS, Beijing, China) at 1:100 dilutions for $60 \mathrm{~min}$ at $37{ }^{\circ} \mathrm{C}$. For negative controls, the primary antibody was replaced with rabbit serum. The standard streptavidin-biotin-peroxidase complex method was performed to detect the primary antibody with the use of a Histostain-Plus Kit (BIOS, Beijing, China). Sections were rinsed in phosphate-buffered saline for $10 \mathrm{~min}$, processed with a Diaminobenzidine Substrate-Chromogen Kit (BIOS, Beijing, China), counterstained in Meyer's hematoxylin, and covered with a neutral balsam-mounting medium. Sections were examined by light microscopy (Olympus, Osaka, Japan). Five sections, at least $25 \mu \mathrm{m}$ apart, were evaluated from each animal (7-9 animals in each group). Following thorough examination of the section (400× magnification), staining was considered to be intense when $50 \%$ or more of the cells were stained. The stained cells in the periodontal ligament were counted with a computer-assisted image-analysis system (Image-pro Plus 6.0, Media Cybernetics, Silver Spring, MD, USA, 2006). The total number of stained cells found in five sections from each animal was used for statistical analysis.

\subsection{Histological Observation of Periodontal Tissues}

Histological observation was conducted following methods described in Han et al. [19] Maxillae with three upper molars were immersed in fixative composed of $4 \%$ paraformaldehyde in phosphate-buffered saline for $4 \mathrm{~h}$ at $4{ }^{\circ} \mathrm{C}$. After the fixation, the specimens were demineralized in $10 \%$ EDTA for 3 weeks. The solution was changed every 2-3 days. The demineralized specimens were then dehydrated in a graded series of ethanol and embedded in $60-62{ }^{\circ} \mathrm{C}$ paraffin. The paraffin sections measuring $5 \mu \mathrm{m}$ in thickness were cut and stained with hematoxylin and eosin. All slides were comparatively evaluated using an Olympus BH2 light microscope (40× and 100×) (Olympus, Osaka, Japan).

\subsection{Morphometric Bone Observation}

Morphometric bone observation was conducted following methods described in Han et al. [19]. The mandibles with three lower molars were defleshed and stained with $1 \%$ methylene blue to delineate the cementoenamel junction. The buccal and lingual surfaces were digitally imaged using a calibrated dissecting microscope $(30 \times)$ (Nikon, New York, NY, USA). The captured images were then further analyzed using a computer-assisted image analysis system (Image-pro Plus 6.0, Media Cybernetics, 
Silver Spring, MD, USA, 2006) by an independent investigator who was unaware of the treatment regimes. The height of alveolar bone loss was calculated as the sum of the exposed molar root area (from the cementoenamel junction to the alveolar bone crest) for both buccal and lingual aspects from the left and right sides.

\subsection{Enzyme-Linked Immunosorbent Assay (ELISA) for Inflammatory Mediators}

ELISA was conducted following methods described in Han et al. [19]. ELISA kits for interleukin-1 $\beta$ (IL-1 $\beta$ ) and tumor necrosis factor- $\alpha(\mathrm{TNF}-\alpha)$ were purchased from R \& D Systems, USA. Two additional ELISA kits for cyclooxygenase (COX-2) and prostaglandin E2 (PGE2) were purchased from Invitrogen (Minneapolis, MN, USA). All assays were performed according to the manufacturer's instructions. Briefly, 96-well plates were separately coated with the antibodies and blocked. Gingiva of mandibular incisor teeth were homogenized on ice in RIPA Lysis Buffer (Beyotime, Jiangsu, China) followed by centrifugation at $12,000 \times g$ at $4{ }^{\circ} \mathrm{C}$. Supernatants were loaded into the wells and incubated at $37^{\circ} \mathrm{C}$ for $60 \mathrm{~min}$. After washing, the plates were incubated with biotinylated antibodies. Plates were then washed four times followed by alkaline phosphatase treatment. After washing the plate, substrate was added to each well for 15 min to allow color development. The reaction was stopped by adding $50 \mu \mathrm{L} 0.5 \mathrm{M} \mathrm{H}_{2} \mathrm{SO}_{4}$ to each well. Absorbance was read at $450 \mathrm{~nm}$ (Tecan GENios, Port Melbourne, Victoria, Austria). Concentrations were normalized to the loaded amount of wet tissue. All experiments were conducted in triplicate.

\subsection{Statistical Analysis}

One-factor ANOVAs were performed to analyze differences in the number of osteoclasts in the periodontal ligament, the area of root exposure, and the inflammatory mediators COX-2 and PGE2 in periodontal tissue, the hippocampus, and cortex. $p$-values $<0.05$ were considered significant.

\section{Conclusions}

Our study provided further evidence for the impact of $\mathrm{AD}$ on periodontal tissues. The forebrain-specific $P S 1 / P S 2$ conditional double knockout in mice led to abnormalities in periodontal tissues at the age of nine and 12 months. Ibuprofen, as a commonly used nonprescription NSAID associated with reduced $\mathrm{AD}$ and periodontitis risk in epidemiological studies, not only improved the abnormalities in periodontal tissues but also attenuated inflammatory responses in the brain and periodontal tissue in $\mathrm{dKO}$ mice at both nine and 12 months. These effects were most likely mediated by the inflammatory cyclooxygenase pathway. The improvement in periodontal tissue abnormalities resulting from ibuprofen treatment could be helpful for oral health maintenance, as well as slow down the progression of periodontal disease due to brain inflammation. 


\section{Acknowledgements}

We deeply appreciate the generosity of Joe Tsien (Medical College of Georgia, USA) for providing PS1/PS2 double knockout mice. This work was supported by grants from the National Natural Science Foundation of China (30840031, 30970726 and 81070876), the Nanometre Science and Technology Project of Shanghai (11nm0504300) and the Shanghai Municipal Education Commission (11ZZ38), and Specialized Research Fund for the Doctoral Program of Higher Education (20110072110041) and was supported, in part, by the 973 Project (2009CB918402).

\section{Conflicts of Interest}

The authors declare no conflict of interest.

\section{References}

1. Hutton, M.; Hardy, J. The presenilins and Alzheimer's disease. Hum. Mol. Genet. 1997, 6, 1639-1646.

2. Feng, R.; Rampon, C.; Tang, Y.P.; Shrom, D.; Jin, J.; Kyin, M.; Sopher, B.; Miller, M.W.; Ware, C.B.; Martin, G.M.; et al. Deficient neurogenesis in forebrain-specific presenilin-1 knockout mice is associated with reduced clearance of hippocampal memory traces. Neuron 2001, 32, 911-926.

3. Donoviel, D.B.; Hadjantonakis, A.K.; Ikeda, M.; Zheng, H.; Hyslop, P.S.; Bernstein, A. Mice lacking both presenilin genes exhibit early embryonic patterning defects. Genes Dev. 1999, 13, 2801-2810.

4. Shen, J.; Kelleher, R.J., 3rd. The presenilin hypothesis of Alzheimer's disease: Evidence for a loss-of-function pathogenic mechanism. Proc. Natl. Acad. Sci. USA 2007, 104, 403-409.

5. Saura, C.A.; Choi, S.Y.; Beglopoulos, V.; Malkani, S.; Zhang, D.; Shankaranarayana Rao, B.S.; Chattarji, S.; Kelleher, R.J., 3rd; Kandel, E.R.; Duff, K.; et al. Loss of presenilin function causes impairments of memory and synaptic plasticity followed by age-dependent neurodegeneration. Neuron 2004, 42, 23-36.

6. Feng, R.; Wang, H.; Wang, J.; Shrom, D.; Zeng, X.; Tsien, J.Z. Forebrain degeneration and ventricle enlargement caused by double knockout of Alzheimer's presenilin-1 and presenilin-2. Proc. Natl. Acad. Sci. USA 2004, 101, 8162-8167.

7. Jiang, X.; Zhang, D.; Shi, J.; Chen, Y.; Zhang, P.; Mei, B. Increased inflammatory response both in brain and in periphery in presenilin 1 and presenilin 2 conditional double knock-out mice. J. Alzheimer's Dis. 2009, 18, 515-523.

8. Beglopoulos, V.; Sun, X.; Saura, C.A.; Lemere, C.A.; Kim, R.D.; Shen, J. Reduced beta-amyloid production and increased inflammatory responses in presenilin conditional knock-out mice. J. Biol.Chem. 2004, 279, 46907-46914.

9. Wang, D.; Yang, L.; Su, J.; Niu, Y.; Lei, X.; Xiong, J.; Cao, X.; Hu, Y.; Mei, B.; Hu, J.F. Attenuation of neurodegenerative phenotypes in Alzheimer-like presenilin 1/presenilin 2 conditional double knockout mice by EUK1001, a promising derivative of xanomeline. Biochem. Biophys. Res. Comm. 2011, 410, 229-234. 
10. Armitage, G.C. Periodontal diagnoses and classification of periodontal diseases. Periodontology 2000 2004, 34, 9-21.

11. Ebersole, J.L.; Cappelli, D.; Mathys, E.C.; Steffen, M.J.; Singer, R.E.; Montgomery, M.; Mott, G.E.; Novak, M.J. Periodontitis in humans and non-human primates: Oral-systemic linkage inducing acute phase proteins. Ann. Periodontol. 2002, 7, 102-111.

12. Kamer, A.R.; Morse, D.E.; Holm-Pedersen, P.; Mortensen, E.L.; Avlund, K. Periodontal inflammation in relation to cognitive function in an older adult Danish population. J. Alzheimer's Dis. 2012, 28, 613-624.

13. Stewart, R.; Sabbah, W.; Tsakos, G.; D’Aiuto, F.; Watt, R.G. Oral health and cognitive function in the Third National Health and Nutrition Examination Survey (NHANES III). Psychosom. Med. 2008, 70, 936-941.

14. Grabe, H.J.; Schwahn, C.; Volzke, H.; Spitzer, C.; Freyberger, H.J.; John, U.; Mundt, T.; Biffar, R.; Kocher, T. Tooth loss and cognitive impairment. J. Clin. Periodontol. 2009, 36, 550-557.

15. Kaye, E.K.; Valencia, A.; Baba, N.; Spiro, A., 3rd; Dietrich, T.; Garcia, R.I. Tooth loss and periodontal disease predict poor cognitive function in older men. J. Am. Geriatr. Soc. 2010, 58, 713-718.

16. Ellefsen, B.; Holm-Pedersen, P.; Morse, D.E.; Schroll, M.; Andersen, B.B.; Waldemar, G. Assessing caries increments in elderly patients with and without dementia: A one-year follow-up study. J. Am. Dent. Assoc. 2009, 140, 1392-1400.

17. Avlund, K.; Holm-Pedersen, P.; Morse, D.E.; Viitanen, M.; Winblad, B. Tooth loss and caries prevalence in very old Swedish people: The relationship to cognitive function and functional ability. Gerodontology 2004, 21, 17-26.

18. Ellefsen, B.; Holm-Pedersen, P.; Morse, D.E.; Schroll, M.; Andersen, B.B.; Waldemar, G. Caries prevalence in older persons with and without dementia. J. Am. Geriatr. Soc. 2008, 56, 59-67.

19. Han, W.; Ji, T.; Wang, L.; Yan, L.; Wang, H.; Luo, Z.; Mei, B.; Su, J. Abnormalities in periodontal and salivary tissues in conditional presenilin 1 and presenilin 2 double knockout mice. Mol. Cell. Biochem. 2011, 347, 13-20.

20. McGeer, P.L.; Schulzer, M.; McGeer, E.G. Arthritis and anti-inflammatory agents as possible protective factors for Alzheimer's disease: A review of 17 epidemiologic studies. Neurology 1996, 47, 425-432.

21. Stewart, W.F.; Kawas, C.; Corrada, M.; Metter, E.J. Risk of Alzheimer's disease and duration of NSAID use. Neurology 1997, 48, 626-632.

22. Vlad, S.C.; Miller, D.R.; Kowall, N.W.; Felson, D.T. Protective effects of NSAIDs on the development of Alzheimer disease. Neurology 2008, 70, 1672-1677.

23. Howell, T.H.; Williams, R.C. Nonsteroidal antiinflammatory drugs as inhibitors of periodontal disease progression. Crit. Rev. Oral Biol. Med. 1993, 4, 177-196.

24. Lim, G.P.; Yang, F.; Chu, T.; Chen, P.; Beech, W.; Teter, B.; Tran, T.; Ubeda, O.; Ashe, K.H.; Frautschy, S.A.; et al. Ibuprofen suppresses plaque pathology and inflammation in a mouse model for Alzheimer's disease. J. Neurosci. 2000, 20, 5709-5714.

25. Lim, G.P.; Yang, F.; Chu, T.; Gahtan, E.; Ubeda, O.; Beech, W.; Overmier, J.B.; Hsiao-Ashec, K.; Frautschy, S.A.; Cole, G.M. Ibuprofen effects on Alzheimer pathology and open field activity in APPsw transgenic mice. Neurobiol. Aging 2001, 22, 983-991. 
26. Cochran, D.L. Inflammation and bone loss in periodontal disease. J. Periodontol. 2008, 79, 1569-1576.

27. Yamamoto, T.; Domon, T.; Takahashi, S.; Islam, N.; Suzuki, R.; Wakita, M. The structure and function of periodontal ligament cells in acellular cementum in rat molars. Ann. Anat. 1998, 180, 519-522.

28. Noble, J.M.; Scarmeas, N. (Cognitive Impairment) Improving Oral Health for the Elderly, 1st ed.; Springer Science \& Business Media: New York, NY, USA, 2008; pp. 99-126.

29. Mei, B. East China Normal University, Shanghai, China. Unpublished observation, 2013.

30. Stalder, A.K.; Carson, M.J.; Pagenstecher, A.; Asensio, V.C.; Kincaid, C.; Benedict, M.; Powell, H.C.; Masliah, E.; Campbell, I.L. Late-onset chronic inflammatory encephalopathy in immune-competent and severe combined immune-deficient (SCID) mice with astrocyte-targeted expression of tumor necrosis factor. Am. J. Pathol. 1998, 153, 767-783.

31. Yang, Y.; Quitschke, W.W.; Brewer, G.J. Upregulation of amyloid precursor protein gene promoter in rat primary hippocampal neurons by phorbol ester, IL-1 and retinoic acid, but not by reactive oxygen species. Brain Res. 1998, 60, 40-49.

32. Li, Y.; Liu, L.; Barger, S.W.; Griffin, W.S. Interleukin-1 mediates pathological effects of microglia on tau phosphorylation and on synaptophysin synthesis in cortical neurons through a p38-MAPK pathway. J. Neurosci. 2003, 23, 1605-1611.

33. In't Veld, B.A.; Ruitenberg, A.; Hofman, A.; Launer, L.J.; van Duijn, C.M.; Stijnen, T.; Breteler, M.M.; Stricker, B.H. Nonsteroidal antiinflammatory drugs and the risk of Alzheimer's disease. N. Eng. J. Med. 2001, 345, 1515-1521.

34. Simmons, D.L.; Botting, R.M.; Hla, T. Cyclooxygenase isozymes: The biology of prostaglandin synthesis and inhibition. Pharmacol. Rev. 2004, 56, 387-437.

35. McGeer, P.L. Cyclo-oxygenase-2 inhibitors: Rationale and therapeutic potential for Alzheimer's disease. Drugs Aging 2000, 17, 1-11.

36. Heneka, M.T.; Sastre, M.; Dumitrescu-Ozimek, L.; Hanke, A.; Dewachter, I.; Kuiperi, C.; O'Banion, K.; Klockgether, T.; van Leuven, F.; Landreth, G.E. Acute treatment with the PPARgamma agonist pioglitazone and ibuprofen reduces glial inflammation and Abeta1-42 levels in APPV717I transgenic mice. Brain 2005, 128, 1442-1453.

37. Hoozemans, J.J.; Veerhuis, R.; Rozemuller, A.J.; Eikelenboom, P. Non-steroidal anti-inflammatory drugs and cyclooxygenase in Alzheimer's disease. Curr. Drug Targets 2003, 4, 461-468.

38. Akiyama, H.; Barger, S.; Barnum, S.; Bradt, B.; Bauer, J.; Cole, G.M.; Cooper, N.R.; Eikelenboom, P.; Emmerling, M.; Fiebich, B.L.; et al. Inflammation and Alzheimer's disease. Neurobiol. Aging 2000, 21, 383-421.

39. Dong, S.; Li, C.; Wu, P.; Tsien, J.Z.; Hu, Y. Environment enrichment rescues the neurodegenerative phenotypes in presenilins-deficient mice. Eur. J. Neurosci. 2007, 26, 101-112.

(C) 2013 by the authors; licensee MDPI, Basel, Switzerland. This article is an open access article distributed under the terms and conditions of the Creative Commons Attribution license (http://creativecommons.org/licenses/by/3.0/). 